\title{
Implicancias Paleoclimáticas y Biogeográficas de Moluscos Terrestres y Dulceacuícolas del Holoceno Tardío en Isla Mocha, Provincia de Arauco, Chile
}

\section{Paleoclimatic and Biogeographic Implications of Late Holocenic Terrestrial and Fresh- Water Mollusks from Isla Mocha, Arauco Province, Chile}

\author{
Donald Jackson ${ }^{1}$, Roberto CampbelL ${ }^{1}$, Constanza Roa ${ }^{1} \&$ Douglas Jackson ${ }^{2}$ \\ ${ }^{1}$ Departamento de Antropología, Facultad de Ciencias Sociales, Universidad de Chile. \\ ${ }^{2}$ Sociedad Chilena de Malacología. \\ *E-mail: djackson@uchile
}

\begin{abstract}
RESUMEN
Evidencia estratigráfica de moluscos terrestres (Endodontidae) y dulceacuícolas (Sphaeridae, Amnicolidae y Succineidae) procedente de contextos arqueológicos de la Isla Mocha, sugieren una marcada tendencia a condiciones más húmedas hacia finales del Holoceno Tardío (post-900 A.P.), en coherencia con antecedentes polínicos previamente conocidos. Estos moluscos proporcionan relevante información para discutir el proceso colonizador de micro-moluscos en esta Isla.
\end{abstract}

Palabras Claves: Arqueología, Biogeografía, Isla Mocha, Moluscos, Paleoclima.

\begin{abstract}
Archaeological excavations on Mocha Island yielded stratigraphic evidence for terrestrial (Endodontidae), and freshwater (Sphaeridae, Amnicolidae, Succineidae) mollusks. It suggests wet conditions by the end of the Late Holocene (post-900 $\mathrm{BP}$ ), what coincide with previous palinological studies. These mollusks provide relevant information to discuss the micromollusks colonization process in this island.
\end{abstract}

KeYwords: Archaeology, Biogeography, Mocha Island, Mollusks, Paleoclimate.

\section{INTRODUCCIÓN}

Las islas son particularmente relevantes para el estudio de procesos biogeográficos y evolutivos dado su aislamiento continental. Es frecuente que la fauna y flora de las islas constituyan biotas diferentes a las continentales, lo que se debe básicamente a su distancia geográfica y al tiempo transcurrido desde su separación (Pefaur \& Yánez 1980). Estos procesos de aislamiento han ocurrido por cambios globales en los niveles marinos (transgresiones) como también por procesos tectónicos locales.

En este marco la Isla Mocha presenta una biota semejante a la valdiviana, planteándose que el aislamiento no fue total y que existió un flujo migratorio permanente que habría impedido la formación de especies propias (Pefaur \& Yánez 1980). Las únicas especies endémicas de la isla serían el anfibio Eupsophus insularis (Phillipi 1902) y el roedor
Octodon pacificus (Hutterer 1994). En esta perspectiva, se ha planteado, por una parte, que la isla habría formado parte del continente y posteriormente habría ocurrido su aislamiento, generándose un poblamiento regresivo donde las especies actuales serían relictos de una fauna mayor, y por otra, que la isla no tuvo contacto con el continente y su poblamiento habría ocurrido por colonización, resultado de deriva marina (vertebrados) y aérea (invertebrados).

La Isla Mocha se ubica a $35 \mathrm{~km}$. de la costa frente a la provincia de Arauco ( $38^{\circ} 22^{\prime} \mathrm{S}, 73^{\circ} 55^{\prime} \mathrm{W}$ ) y tiene un extensión de $52 \mathrm{~km}^{2}$ (Pefaur \& Yánez 1980). Geológicamente está constituida por un complejo de edad miocénica (Tavera \& Veyl 1958), presentando una topografía montañosa formada por dos cordones paralelos a la costa con alturas que alcanzan los 390 m.s.n.m. y una serie de terrazas de origen marino (Prieto 1997). Otros estudios geológicos muestran que desde los 6000 años es activa tectónicamente, 
con levantamientos cosísmicos (terremotos de 1835 y 1960) y asísmico provocado por el desplazamiento en una falla inversa de la Placa Sudamericana (Nelson \& Manley 1992).

Climáticamente está caracterizada por una fuerte influencia oceánica con temperaturas mínimas y máximas medias anuales de alrededor de $9,4^{\circ}$ y $15,5^{\circ}$, humedad promedio cercana al $85 \%$ y precipitaciones promedio de 1317 mm (Hajek \& Di Castri 1975). Se han distinguido cuatro formaciones vegetacionales destacando el bosque (Pefaur \& Yánez 1980), especialmente de olivillo que se caracteriza por la predominancia de especies arbóreas ornitócoras (Aextoxicon punctatun, Drymys winteri, Luma apiculata, Myrceugenia planipes entre otras), con notoria ausencia de taxas arbóreas del tipo de bosque templado-lluvioso de Chile, como algunas especies de fagáceas y proteáceas (Lequesne et al. 1999).

Muestreos faunísticos atestiguan la presencia de cuatro grupos de vertebrados, constituidos por cuatro especies de anfibios, dos especies de reptiles y cinco especies de mamíferos, además de aproximadamente 102 especies de aves (Reiche 1903; Pefaur \& Yánez 1980).

Los estudios arqueológicos han indicado la existencia de alrededor de cuarenta asentamientos indígenas, tanto prehispánicos como históricos (Sánchez 1997; Quiroz 2003). Los sitios arqueológicos más antiguos corresponden a una acotada ocupación correspondiente al Holoceno MedioTardío datada alrededor de 3400 A.P. (o 1500 a.C.) (Vásquez 1997; Quiroz \& Sánchez 2004). Tras está ocupación parece haber un hiato ocupacional que se extiende hasta alrededor del 600 d.C. Desde esa fecha aparecen contextos adscritos a poblaciones portadoras de cerámica (Sánchez et al. 2004; Quiroz \& Sánchez 2005), y que perduran hasta el 1687 d.C., momento en que Isla Mocha es despoblada. Esta última condición se mantiene hasta la década de 1850 cuando se inicia el repoblamiento que da origen a la población actual.

En este contexto, la presencia de moluscos terrestres y dulceacuícolas en Isla Mocha, proporciona algunas variables relacionadas con su paleoclima y biogeografía. Por otra parte su frecuencia sugiere cambios de humedad y proporciona valiosa información para discutir su colonización como resultado del transporte activo (deriva aérea) y transporte pasivo (deriva marina), así como por agentes antrópicos, ocurridos durante distintos momentos del Holoceno.

\section{MATERIALES Y MÉTODOS}

Recientes excavaciones estratigráficas (Campbell 2011) realizadas en el sitio arqueológico Parcela 29-1 (P291) han permitido la recuperación y estudio de moluscos terrestres, anfibios y dulceacuícolas de data prehispánica, y posiblemente histórica (post-1550 d.C.). Este sitio se ubica al noreste de Isla Mocha, aproximadamente a $1 \mathrm{~km}$ de la costa actual y a una altura de $30 \mathrm{msnm}$. El sitio corresponde a un asentamiento doméstico con una extensión aproximada de 9 hectáreas. Se encuentra disturbado en sus niveles más superficiales (hasta alrededor de los $30 \mathrm{~cm}$ de profundidad) por la actividad agrícola. Hacia el extremo suroeste el sitio limita con una pequeña laguna de agua dulce de unos $200 \mathrm{~m}^{2}$.

La excavación estratigráfica del sitio muestra básicamente tres capas desde abajo hacia arriba: I. Depósito de una matriz de limo pardo oscura con restos de moluscos marinos y carbón resultado de una ocupación humana, la que se extiende desde los 85 a $50 \mathrm{~cm}$. de profundidad, II. Depósito de una matriz limo-arcillosa de color negro correspondiente a un fogón lenticular de origen cultural, con un espesor que va desde los 50 a $40 \mathrm{~cm}$. de profundidad y, III. Depósito de matriz franco limo-arcillosa con restos de conchilla de color gris oscuro que abarca desde los $40 \mathrm{~cm}$. de profundidad a la superficie actual.

Como parte de la excavación se realizó una columna de flotación aledaña al pozo de sondeo, obteniendo 16 muestras de suelo de 6.13 litros c/u., recuperadas cada 5 $\mathrm{cm}$. Estas muestras fueron flotadas separando la fracción pesada de la liviana. En la fracción liviana se separaron los restos orgánicos (carporestos vegetales), discriminando los micro-moluscos terrestres y dulceacuícolas. Los moluscos, una vez separados bajo lupa binocular (80x), fueron identificados taxonómicamente a nivel de género, siguiendo los criterios de Biese (1947), Stuardo (1961), Valdovinos \& Stuardo (1989), Vargas-Almodacid (2000) e Ituarte (2007). La distribución geográfica se asignó según Valdovinos (1999, 2006) y Parada \& Peredo (2006). Posteriormente, se cuantificaron por grupos taxonómicos y distribución estratigráfica, discutiendo su frecuencia en relación a otros restos orgánicos recuperados constituidos por carporestos vegetales (Roa 2011) y sobre la base de los antecedentes polínicos conocidos para la isla (Lequesne et al. 1999).

\section{RESULTADOS}

El análisis de las 16 muestras de la fracción liviana, permitió recuperar un total de 84 micro-moluscos, entre los cuales se encuentra una especie humícola del género Radiodiscus (Endodontidae), una especie anfibia, Succinea sp., que probablemente corresponda a Succinea chiloensis (Succineidae) y dos especies dulceacuícolas, que incluyen un bivalvo de la familia Sphaeridae (Pisidium sp.) y un gastrópodo de la familia Amnicolidae (Littoridina sp.). 
La cuantificación por grupos taxonómicos (Fig. 1), muestra un predominio de Radiodiscus sp. (Endodontidae) con un total de 58 (69.05\%) ejemplares, seguido por Succinea $\mathrm{sp.}$ (Succineidae) con 19 (22.62\%) ejemplares, mientras que Pisidium sp. (Sphaeridae) se encuentra representado por 4 (4.76\%) ejemplares y Littoridina sp. (Amnicolidae) sólo por $3(3.57 \%)$ especímenes.

Por otra parte, si se subdivide la secuencia estratigráfica en dos bloques (Fig. 2); niveles superiores (0-40 cm.) y niveles inferiores (40-80 cm), la distribución en el perfil, muestra un predominio de los moluscos en los niveles superiores (76.2\%) respecto a los inferiores (23.8\%). A su vez, en los niveles superiores predominan los moluscos terrestres (humícolas) como Radiodiscus sp. (78.8\%) sobre las especies de ambientes acuáticos (Succineidae, Sphaeridae y Amnicolidae) (28.2\%). En cambio, para los niveles inferiores la relación entre moluscos terrestres y acuáticos es relativamente proporcional (60\% vs $40 \%)$.
Esta tendencia sugiere que las condiciones locales variaron temporalmente desde más húmedas (presencia de agua) a menos húmeda. Probablemente cercano al sitio existió un bofedal o un ambiente acuático somero, talvez el mismo que observamos hoy cercano al sitio, el que fue disminuyendo en su nivel freático al mismo tiempo de permitir el florecimiento de vegetación arbórea, la que generó un sustrato humícola para el desarrollo de especies como Radiodiscus sp.

La obtención de tres dataciones radiocarbónicas (AMS) sobre carbones (Tabla 1) permite situar cronológicamente estos eventos. Pese a que los resultados muestran una inversión cronológica (la que no se condice con el aspecto intacto de la estratigrafía sub-30 cm de profundidad), estos pueden ser considerados relativamente contemporáneos en un lapso temporal que cubre aproximadamente 350 años, entre los 1049 a 1384 años D.C. Adicionalmente, las evidencias arqueológicas (Campbell 2011) del sitio tienden a indicar que tras esta última fecha (1384 D.C.) este asentamiento continuó siendo ocupado.

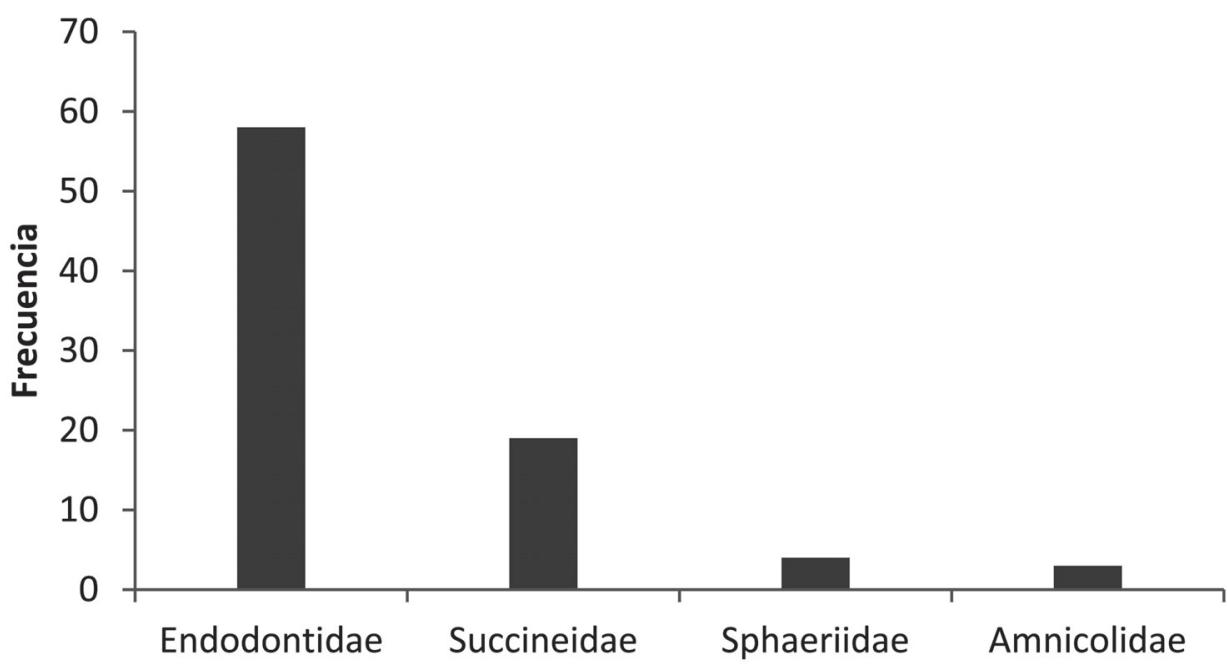

FIGURA 1. Frecuencia absoluta de moluscos por grupos taxonómico.

FIGURE 1. Mollusks absolute frequency by taxon groups.

Tabla 1. Dataciones Radiocarbónicas de Sitio P29-1, Isla Mocha (calibradas en el programa Calib 6.0 [Stuiver \& Reimer 1993; Stuiver et al. 2005] usando la curva SHCal04 [McCormac et al. 2004]).

TABLE 1. Radiocarbon dates from P29-1 site, Isla Mocha (calibrated in the Calib 6.0 program [Stuiver \& Reimer 1993; Stuiver et al. 2005] using the SHCal04 curve [McCormac et al. 2004]).

\begin{tabular}{l|c|c|c|c|c|c}
\hline Código Laboratorio & Profun-didad & Fecha ${ }^{14} \mathrm{C}$ & $\mathrm{d}^{13} \mathrm{C}$ & $\begin{array}{c}\text { Fecha Calibrada A.P. } \\
2 \text { sigmas }\end{array}$ & $\begin{array}{c}\text { Fecha Calibrada } \\
\text { d.C. 2 sigmas }\end{array}$ & Material \\
\hline AA 89416 & $30-40 \mathrm{~cm}$. & $895 \pm 38$ & -26.4 & $901-680$ & $1049-1270$ & Carbón \\
AA 89417 & $50-60 \mathrm{~cm}$. & $825 \pm 36$ & -25.0 & $761-663$ & $1189-1287$ & Carbón \\
AA 89418 & $70-80 \mathrm{~cm}$. & $759 \pm 38$ & -25.6 & $724-566$ & $1226-1384$ & Carbón \\
\hline
\end{tabular}




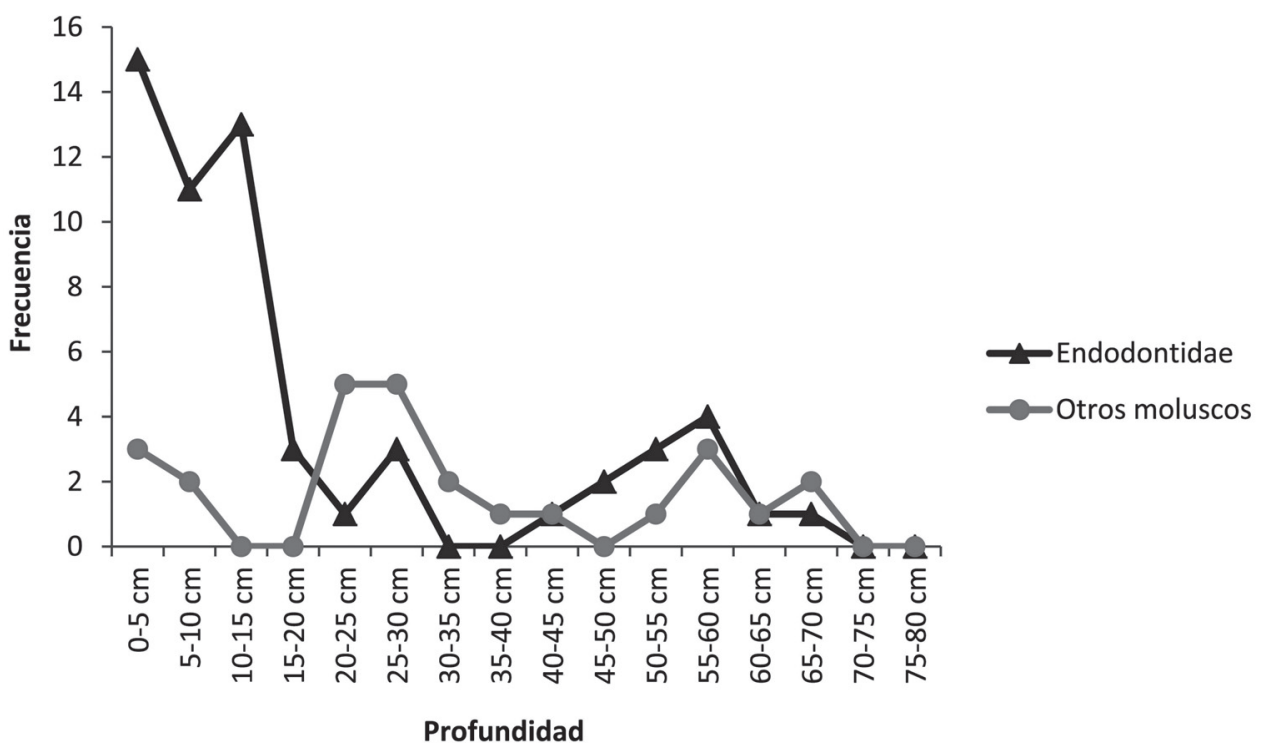

Figura 2. Distribución estratigráfica de moluscos terrestres (Endodontidae) y otros moluscos dulceacuícolas (Succineidae, Sphaeridae y Amnicolidae).

Figure 2. Stratigraphic distribution of terrestrial (Endodontidae) and other fresh-water (Succineidae, Sphaeridae, and Amnicolidae) mollusks.

Si bien estos resultados se basan en un único pozo de sondeo y la frecuencia de moluscos es escasa, muestran una relativa concordancia con los resultados de columnas polínicas realizadas en la isla (Lequesne et al. 1999).

Por otra parte, se sabe de la presencia de otras especies de moluscos procedentes de contextos arqueológicos de la isla. En este sentido, en el sitio P5-1 se ha constatado la presencia de Diplodon sp. (Gálvez 1997), en un contexto datado hacia $\operatorname{los} 740 \pm 100$ años A.P. y los $1210 \pm 110$ años A.P. (Sánchez 1997), mientras que en los niveles superiores del sitio P271 se ha registrado Plestostylus sp. (Gálvez 1997), datado hacia $\operatorname{los} 3220 \pm 50$ años A.P.

Estos antecedentes atestiguan la presencia de al menos seis géneros de moluscos (2 terrestres, 3 dulceacuícolas y 1 anfibio), con dataciones prehispánicas para la isla, todos los cuales pueden asociarse a nivel genérico con la fauna de moluscos existentes para la misma latitud pero en el continente (Parada \& Peredo 2006; Valdovinos 1999, 2006; Valdovinos \& Stuardo 1988), sugiriendo la ausencia de endemismo para estos invertebrados, como ocurre con prácticamente todos los vertebrados tetrápodos de la isla (Pefaur \& Yañez 1980).

\section{DISCUSIÓN}

Los cambios observados en la frecuencia de moluscos del sitio P-29-1, datados entre los $759 \pm 38$ años A.P. y los 895 \pm 38 años A.P., serían coincidentes con algunas de las fases húmedas detectadas a partir del análisis de las asociaciones de polen fósil, obtenidas de dos columnas polínicas realizadas en Laguna Hermosa y Laguna Huairavos de Isla Mocha. Allí se sugiere el mantenimiento del bosque durante los pasados 2000 años. No obstante, los registros indican que el bosque de "olivillo" (Aextoxicon punctatum) y otras Mirtáceas se han visto interrumpidas por varias fases húmedas con dominio de bosques de "canelo" (Drimys winteri), lo que se ha asociado a terrenos anegados o saturados de agua, dado la presencia de herbáceas palustres y helechos (Lequesne et al. 1999).

Las dataciones indicarían que estos cambios ocurrieron a una escala centenaria, dadas las relativas coincidencias con los diagramas polínicos, así como por la mayor presencia de especies vegetales acuáticas para la zona (Scirpus sp.) (Roa 2011), mostrando la ocurrencia de fases húmedas hacia finales del Holoceno asociadas con ambientes palustres que albergaron moluscos dulceacuícolas.

La presencia de estos moluscos terrestres y dulceacuícolas en estos ambientes también tienen relevantes implicancia biogeográficas. A este respecto, sabemos que al término del Pleistoceno la isla habría quedado totalmente expuesta hasta una cota de 40 m.s.m.m, como resultado de un fuerte evento de alzamiento (Prieto 2007), lo que habría permitido una temprana colonización de la isla. No obstante lo anterior, es posible también, que la colonización de la isla, ocurriera durante los períodos de transgresiones marinas más tardías, 
datadas hacia los 8.200 a 8.900 años A.P. y 5.500 a 6.100 años A.P. (Nelson \& Manley 1992; Prieto 1997).

Estas transgresiones habrían posibilitado el transporte pasivo por deriva marina desde el continente, a través de troncos donde se habrían adheridos moluscos terrestres. En este sentido, la descarga de ríos como el Tirúa, frente a la isla, debió ser mayor que en la actualidad y probablemente facilitó el transporte de troncos hacia el mar, luego las corrientes oceánicas habrían transportado mar "adentro" estas descargas, como ocurre en la actualidad con las materiales derivados de la actividad antrópica a lo largo de toda la costa chilena (Thiel et al. 2003; Hinojosa \& Thiel 2009).

Después de los 6.000 años A.P., y particularmente con posterioridad a los 2200 años A.P., el alzamiento tectónico de la isla, deja expuesta al menos tres terrazas marinas (Nelson \& Manley 1992; Prieto 1997), sobre las cuales se habrían desarrollado ambientes lagunares y palustres, donde pudieron propagarse los moluscos terrestres, anfibios y dulceacuícolas. Los de agua dulce pudieron llegar a la isla, por deriva aérea, considerando que la isla alberga al menos 102 especies de aves (Pefaur \& Yáñez 1980). La adherencia de estos micro-moluscos o sus huevos a las patas de las aves pudo ser el mecanismo de transporte desde la existencia misma de la isla.

Sin duda la presencia humana, la que ocurriría por primera vez hacia los 1.500 años a.C. (3400 años A.P.) en dos asentamientos de cazadores y recolectores de la isla (Vásquez 1997), marcó un hito crono-cultural para el inicio de un transporte antrópico de distintas especies, primero en forma pasiva y posteriormente de forma intencional.

El transporte pasivo de moluscos pudo ocurrir adherido a plantas comestibles así como en los propios atuendos de estos primeros ocupantes y navegantes. Mientras que, posteriormente con la llegada de poblaciones portadoras de cerámica, el transporte de moluscos como el Diplodon sp. fue intencional para ser consumido por éstas y otras poblaciones (Lara et. al. 1988; Jackson \& Jackson 2008). Este proceso continuo posteriormente, con la llegada de los primero europeos a partir de 1544, con quienes los indígenas de la isla tuvieron un significativo intercambio y luego desde mediados del siglo XIX, con la llegada de los actuales mochanos.

\section{CONCLUSIONES}

Las diferencias de frecuencia de los moluscos terrestres y dulceacuícolas observadas en la secuencia estratigráfica del sitio P29-1, muestran eventos húmedos hacia finales del
Holoceno en coherencia con los registros polínicos de la isla (Lequesne et al. 1999), mostrando las condiciones húmedas zonales y azonales para la isla durante fines del Holoceno Tardío.

Por otra parte, las evidencias sugieren que los moluscos registrados en la isla llegaron por procesos de colonización pasiva o por el transporte antrópico indirecto, lo que pudo ocurrir hacia finales del Pleistoceno cuando la isla quedo expuesta, no obstante esta colonización es probable que comenzara hacia los 8.200 a 8.900 en co-ocurrencia con los más antiguos procesos transgresivos. La colonización pudo generarse por procesos de transporte natural, tanto por deriva marina como aérea, así como por la ocupación humana de la isla que se remonta a unos 3400 años A.P. La colonización ocurrió entonces en distintos momentos y por distintos procesos.

Finalmente, futuros estudios genéticos de moluscos, en poblaciones actuales de la isla y del continente podrían contribuir a modelar y contrastar las hipótesis aquí planteadas.

\section{AGRADECIMIENTOS}

Esta investigación se ha realizado en el marco de los proyectos NSF-BCS 0956229 y FONDECYT 3130515.

\section{BIBLIOGRAFÍA}

BIESE, W. 1947. Revisión de los moluscos terrestres y de agua dulce provistos de concha de Chile. II parte. Fam. Amnicolidae. Boletín del Museo Nacional de Historia Natural 23:67-77.

Campbell, R. 2011. Socioeconomic Differentiation, Leadership, and Residential Patterning at an Araucanian chiefly center (Isla Mocha, AD 1000-1700). Ph.D. Dissertation, University of Pittsburgh, Pittsburgh.

GÁlvez, O. 1997. Análisis de los restos malaco-arqueológicos de la Isla Mocha. En: La Isla de Las Palabras Rotas (Eds. Quiroz, D. \& M. Sánchez), pp. 195-207. Dirección de Bibliotecas, Archivos y Museos y Centro de Investigaciones Diego Barros Arana, Santiago.

Hajek, E. \& F. Di Castri. 1975. Bioclimatografia de Chile. Dirección de Investigación, Vice-Rectoría Académica Universidad Católica de Chile, Santiago. URL: http:// www.ecolyma.cl/documentos/bioclimatografia_de_chile. pdf Accesado: Mayo 17, 2013.

Hinojosa I. \& M. Thiel. 2009. Floating marine debris in fjords, gulfs and channels of southern Chile. Marine Pollution Bulletin 58:341-350.

HutTERER, R. 1994. Island rodents: a new species of Octodon from Isla Mocha, Chile (Mammalia: Octodontidae). Zeitschrift für Säugetierkunde 59:27-51.

Ituarte, C. 2007. Las especies de Pisidium Pfeiffer de Argentina, Bolivia, Chile, Perú y Uruguay (Bivalvia-Sphaeriidae). 
Revista del Museo Argentino de Ciencias Naturales 9(2):169-203.

JACKSON, D. \& D. JACKSON. 2008. Antecedentes arqueológicos para Diplodon (Spix 1827) (Bivalvia, Hyriidae) en Chile. Gayana 72(2):196-203.

Mccormac, F., A. Hogg, P. Blackwell, C. Buck, T. Higham \& P. ReIMER. 2004. SHCal04 Southern Hemisphere Calibration, 0-11.0 cal kyr BP. Radiocarbon 46:1087-1092.

Lara G, E. Parada, S. Peredo, J. Inostroza \& H. Mora. 1988. La almeja de agua dulce Diplodon chilensis (Gray, 1828), Un recurso potencial. Boletín Museo Regional de La Araucanía 3:33-40.

Lequesne C., C. Villagran \& R. Villa. 1999. Historia de los bosques relictos de "olivillo" (Aextoxicon punctatum) y Mirtáceas de la Isla Mocha, Chile, durante el Holoceno Tardío. Revista Chilena de Historia Natural 72:31-47.

Nelson A. \& W. Manley. 1992. Holocene coseismic and aseismic uplift of Isla Mocha south-central Chile. Quaternary International 15-16:61-76.

Parada E. \& S. Peredo. 2006. Estado de conocimiento de los Bivalvos dulceacuícolas de Chile. Gayana 70(1):82-87.

Pefaur, J. \& J. YÁñez. 1980. Ecología Descriptiva de la Isla Mocha (Chile), en Relación al Poblamiento de Vertebrados. Boletín del Museo Nacional de Historia Natural 37:103112.

Philippi, R. A. 1902. Suplemento a los Batraquios chilenos descritos en la Historia Física y Política de Chile de don Claudio Gay. Imprenta de Enrique Blanchard-Chessi, Santiago.

Prieto, X. 1997. Evolución geomorfológica de Isla Mocha durante el Holoceno. En: La Isla de Las Palabras Rotas (Eds. Quiroz, D. \& M. Sánchez), pp. 87-101. Dirección de Bibliotecas, Archivos y Museos y Centro de Investigaciones Diego Barros Arana, Santiago.

Quiroz, D. 1997. Fragmentos recuperados: un breve panorama histórico para la Isla Mocha. En: La Isla de Las Palabras Rotas (Eds. Quiroz, D. \& M. Sánchez), pp. 237-241. Dirección de Bibliotecas, Archivos y Museos y Centro de Investigaciones Diego Barros Arana, Santiago.

Quiroz, D. 2003. Catastro patrimonio arqueológico Mapuche, Provincia de Arauco, Informe Final. Gobierno Regional: Región del Biobio, y Gobierno de Chile: Ministerio de Planificación y Cooperación y Conadi Dirección Regional del Bio-Bio, Santiago y Concepción.

Quiroz, D. \& M. SÁnchez. 2004. Poblamientos iniciales en la costa septentrional de la Araucanía (6.500-2.000 a.p.). Chungará 36 (suplemento especial) Tomo I: 289-302.

Quiroz, D. \& M. SÁNChEz. 2005. La secuencia Pitrén-El Vergel en Isla Mocha: Soluciones de continuidad y distinciones culturales. Actas del XVI Congreso Nacional de Arqueología Chilena, pp. 369-378. Museo de Historia Natural de Concepción y Escaparate Ediciones, Concepción.
Reiche, C. 1903. Reseña zoolójica de la isla. En: La Isla de la Mocha (Ed. Reiche, C.), pp. 46-48. Anales del Museo Nacional de Chile, Santiago.

RoA, C. 2011. Los recursos vegetacionales en el registro arqueológico del Período Alfarero Tardío en Isla Mocha. Informe Práctica Profesional de Arqueología, Departamento de Antropología, Facultad de Ciencias Sociales, Universidad de Chile, Santiago.

SÁnchez, M. 1997. El Período Alfarero en la Isla Mocha. En: La Isla de Las Palabras Rotas (Eds. Quiroz, D. \& M. Sánchez), pp. 103-131. Dirección de Bibliotecas, Archivos y Museos y Centro de Investigaciones Diego Barros Arana, Santiago.

SÁnchez, M., D. Quiroz, \& M. Massone. 2004. Domesticación de Plantas y Animales en la Araucanía: Datos, Metodologías y Problemas. Chungará 36 (suplemento especial) Tomo I: 365-372. Arica.

Stuardo, J. 1961. Contribución a un catálogo de los moluscos Gasterópodos Chilenos de Agua Dulce. Gayana Zoológica 1:7-31.

Stuiver, M., P. Reimer \& R. Reimer. 2005. Calib 6.0. www program and documentation. URL: http://calib.qub.ac.uk/ calib/ Accesado: Mayo 17, 2013.

Stuiver, M. \& P. Reimer. 1993. Extended 14C data base and revised CALIB 3.0 14C age calibration program. Radiocarbon $35: 215-230$.

Tavera J. \& Veyl. 1958. Reconocimiento Geológico de la Isla Mocha. Publicación Departamento de Geología, Universidad de Chile 12:157-188.

Thiel M., I. Hinojosa, N. VÀsquez \& E. Macaya. 2003. Floating marine debris in coastal waters of the SE-Pacific (Chile). Marine Pollution Bulletin 46:224-231.

Vargas-Almonacid, P. 2000. Nueva especie de microgastrópodo humícola para Chile, Radiodiscus quillajicola Spec. Nov. (Stylommatophora: Charopidae). Gayana Zoológica 64:61-66.

Valdovinos, C \& J. Stuardo. 1989. Nuevo gastrópodo húmicola de Chile Austrodiscus (Zilchogyra) solemi Spec. Nov. (Pulmonada: Endodontidae). Boletín de la Sociedad Biológica de Concepción 60:239-245.

Valdovinos, C. 1999. Biodiversidad de Moluscos Chilenos: Base de datos Taxonómica y distribucional. Gayana 63(2):111164.

Valdovinos, C. 2006. Estado de conocimiento de los Gastropodos dulceacuícolas de Chile. Gayana 70(1):88-95.

Valdovinos C. \& J. Stuardo. 1988. Morfología, Sistemática y Distribución del género Plectostylus Beck, 1837 (Pulmonata: Bulimulidae). Gayana 52(1-2):115-195.

Vásquez, M. 1997. El Período Arcaico en Isla Mocha. En: La Isla de Las Palabras Rotas (Eds. Quiroz, D. \& M. Sánchez), pp. 215-235. Dirección de Bibliotecas, Archivos y Museos y Centro de Investigaciones Diego Barros Arana, Santiago.

Recibido:13.12.11

Aceptado: 14.06.13 